\title{
Effectiveness of vitrectomy in Terson syndrome - case series
}

\author{
Edyta Koman', Beata Gajda', Agnieszka Kiszka', Agnieszka Cisek², \\ Katarzyna Nowomiejska', Robert Rejdak' \\ ${ }^{1}$ Department of General Ophthalmology, Medical University of Lublin, Poland \\ ${ }^{2}$ Department of Human Anatomy, Medical Faculty University of Rzeszow, Poland
}

\begin{abstract}
The visual outcomes of vitrectomy in a series of five eyes of three patients with vitreous haemorrhage due to Terson syndrome were retrospectively evaluated. There were two bilateral cases and one unilateral. The neurological diagnosis included cranio-cerebral trauma and cerebral aneurysm rupture. The mean time interval between intracranial haemorrhage and vitrectomy was nine months (range 7-10 months). Pars plana vitrectomy was performed with BSS as a tamponade in three eyes, SF6 gas in one eye, and silicone oil in one eye. The mean follow-up period was 12 months (range 1-27 months). Visual acuity improved significantly in all patients from counting fingers to the mean value of 0.6 (range $0.1-1.0$ ). We observed one epiretinal membrane, one tractional retinal detachment, and two cataract formations. The best visual outcomes were achieved with BSS as a tamponade, the worst was with silicone oil as a tamponade and retinal detachment. Performing vitrectomy with BSS as a tamponade in patients with Terson syndrome significantly improves visual acuity, so early diagnosis and surgical treatment are crucial.
\end{abstract}

KEY WORDS: Terson syndrome, intracranial haemorrhage, vitrectomy

Ophthalmol J 2016; Vol. 1, No. 2, 73-77

\section{INTRODUCTION}

Terson syndrome is defined as a haemorrhage in the eye associated with earlier intracranial bleeding [1]. Intraretinal haemorrhage associated with subarachnoid bleeding was first described by Litten in 1881 , but the case described in 1900 by the French ophthalmologist Albert Terson was named as this syndrome [2-4]. Nowadays, according to this definition, this syndrome comprises all forms of intraocular haemorrhage associated with intracranial bleeding or raised intracranial pressure (ICP). Haemorrhage occurring during the course of Terson syndrome may be present in one or both eyes [2]. Clinical symptoms include blurred vision, which correlates with the severity of bleeding, usually with characteristic intracranial haemorrhage neurological symptoms, such as loss of consciousness and coma. Terson syndrome occurs in $10-20 \%$ of patients who experience subarachnoid haemorrhage, but is less likely to be associated with subdural haematoma or traumatic epidural haematoma or resulting from other causes [5]. Differential diagnosis should include another cause of intraocular bleeding as diabetic retinopathy, age-related macular degeneration (AMD), intraocular tumour, or retinal detachment [2]. Ophthalmic examinations, diagnosis, and possible treatment (mainly surgical) can reduce the likelihood of subsequent visual impairments or loss of vision.

Below we present the results of surgical treatment with vitrectomy of three patients with diagnosed Terson syndrome — two bilateral and one unilateral. 


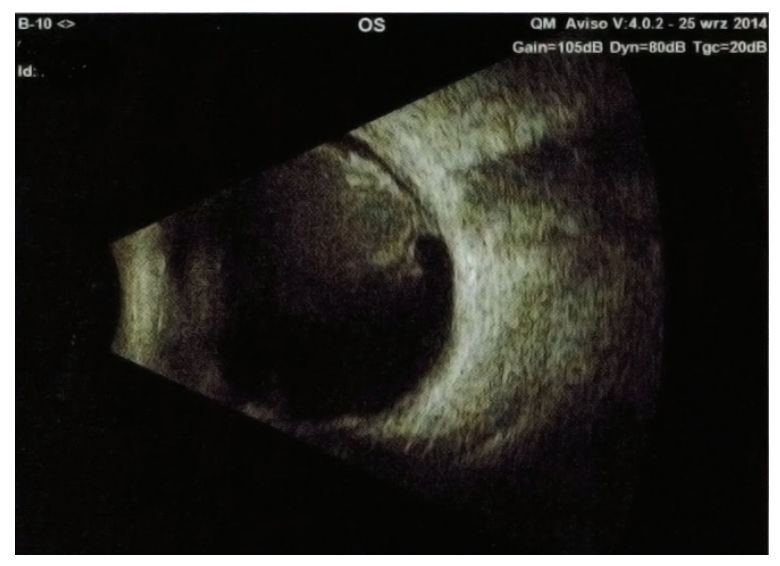

FIGURE 1. Ultrasonography of the eyeball with vitreous haemorrhage in the course of Terson syndrome

\section{CASE 1}

A 33-year-old woman was diagnosed with intraocular bilateral vitreous haemorrhage due to cerebral trauma accompanied with quadriplegia six months previously. On admission to the hospital the best-corrected visual acuity (BCVA) was hand movements in the right eye and counting fingers to one metre in the left eye. Intraocular pressure (ICP) and anterior segment of both eyes were normal. Ultrasonography (USG) of the eyeball confirmed haemorrhage into the vitreous cavity, and a diagnosis of Terson syndrome was made (Fig. 1). Planned surgery under general anaesthesia was delayed because the patient had taken drugs with monoamine oxidase inhibitors. After psychiatric consultation and change of treatment, a month later (eight months after trauma) vitrectomy was performed with balanced salt solution (BSS) as endotamponade on the left eye. BCVA before the surgery was hand movements and on discharge from the hospital it was 0.1. Another control, a month later, showed further improvement in vision of the left eye to 0.4 . One month after vitrectomy with BSS as endotamponade was performed on the left eye, the same procedure was carried out on the right eye. Postoperatively, BCVA was counting 0.2 without correction. Two months after second vitrectomy BCVA was 0.3 in the right eye and 0.8 in the left eye.

\section{CASE 2}

A 48-ear-old man presented with subvitreous haemorrhage in the left eye as a consequence of cerebral aneurysm rupture and stroke. BCVA was a 1.0 in the right eye and counting fingers to one metre in the left eye. Anterior segment and intraocular pressure of both eyes were correct. First, a cataract surgery of the left eye was performed due to poor lens translucency. A month later (10 months after the neurological incident), vitrectomy was done with BSS as endotamponade. Postoperatively, BCVA of the left eye was 0.3 , after two weeks - 0.5, and after a month -0.7 (Figs. 2, 3).

\section{CASE 3}

A 36-year-old man presented with the haemorrhage into the vitreous of both eyes, as a result of cerebral aneurysm rupture. On admission to the hospital, BCVA was counting fingers to 2 metres in both eyes. Seven months after the neurological incident, vitrectomy was performed in the right eye with ILM peeling due to the presence of epiretinal membrane and gas SF6 as endotamponade. The control examination after one month indicated BCVA as 0.8 in the right eye. After three months, a vitrectomy was performed in the left eye with simultaneous intraocular lens implantation due to cataract formation and 5000 cst silicone oil as end tamponade due to the presence of haemorrhage and retinal tractional detachment. Further control after one month brought improvement of BCVA to 0.1 . Due to the presence of proliferative vitreoretinopathy, a year after the operation on the left eye, a second vitrectomy was performed with silicone oil replacement and relaxing retinotomy. The vision of the left eye was still maintained at the level of 0.1 , and IOP is correct. After two years, BCVA of the right eye was 0.7 and on the left eye - counting fingers to four metres.

\section{DISCUSSION}

Intraocular bleeding accompanying cerebral haemorrhage may be subretinal, intraretinal, epiretinal, subvitreal, or intravitreal [3]. The most common form of bleeding is subvitreal haemorrhage associated with subarachnoid haemorrhage $(\mathrm{SAH})$ - 10-50\%. Simultaneous haemorrhages into vitreous and retina occur in about $18-20 \%$ of cases, with the least likely (4\%) being haemorrhage into the vitreous humour only [6]. Most often, this syndrome is described as a correlation of subarachnoid haemorrhages with ruptured cerebral vessel aneurysms, mainly in three locations: in the internal carotid artery, the middle cerebral artery bifurcation, and in the upper part of the basal artery. The anatomical 


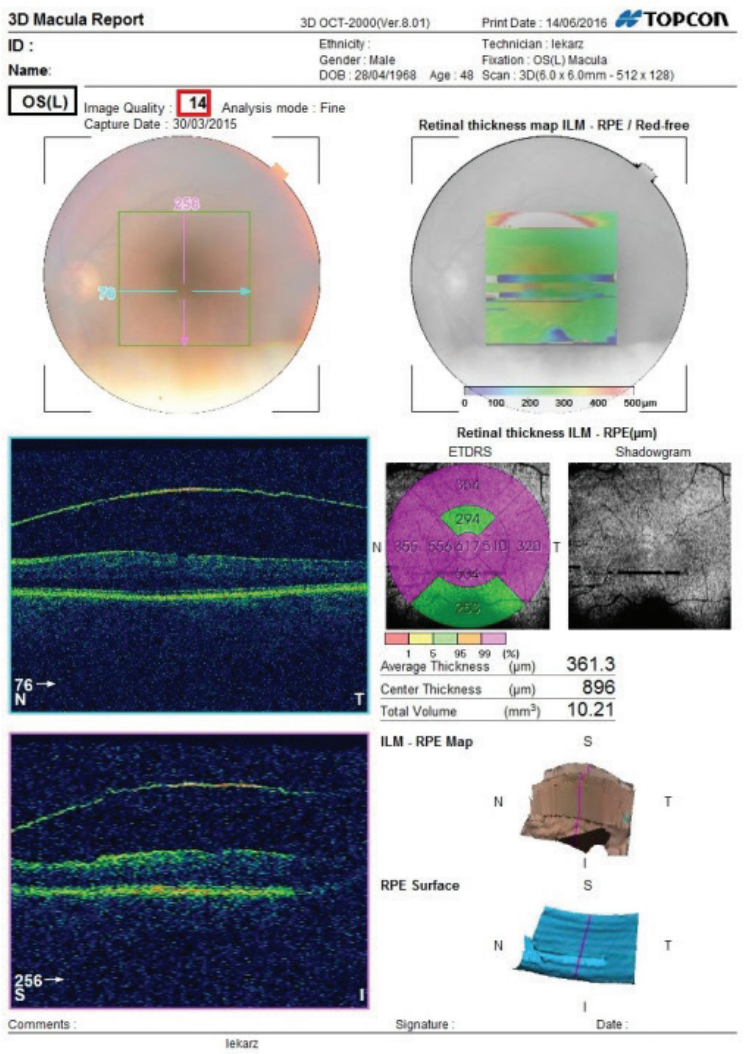

FIGURE 2. Ocular coherence tomography of the macula before vitrectomy in a patient with Terson syndrome

location of the aneurysm (or vascular anomalies) is not associated with the site in which the Terson syndrome appears. It has also been proven that anatomical proximity between the aneurysm and the eyeball is not required for intraocular haemorrhage appearance [7]. In other reports such reasons are described: trauma, hypertension, tumour, and perioperative and postoperative intracranial bleeding [3].

So far, the pathogenesis of Terson syndrome remains unclear. According to the most probable mechanism, the leading cause of bleeding is increased ICP, which causes oedema of the optic nerve. The blood returns from the retinal veins into the cavernous sinus with greater difficulty, and as a result the vessels of the retina and the choroid at the level of plaque sieve become closed. Increased intracranial venous pressure generated in the retinal veins causes stasis and rupturing of superficial retinal vessels, leading to the intraocular haemorrhage. This theory explains the occurrence of Terson syndrome in cases other than subarachnoid haemorrhage $[2,5,8]$.

Diagnostics of Terson syndrome usually begins with extended neurological tests. After neuroim-

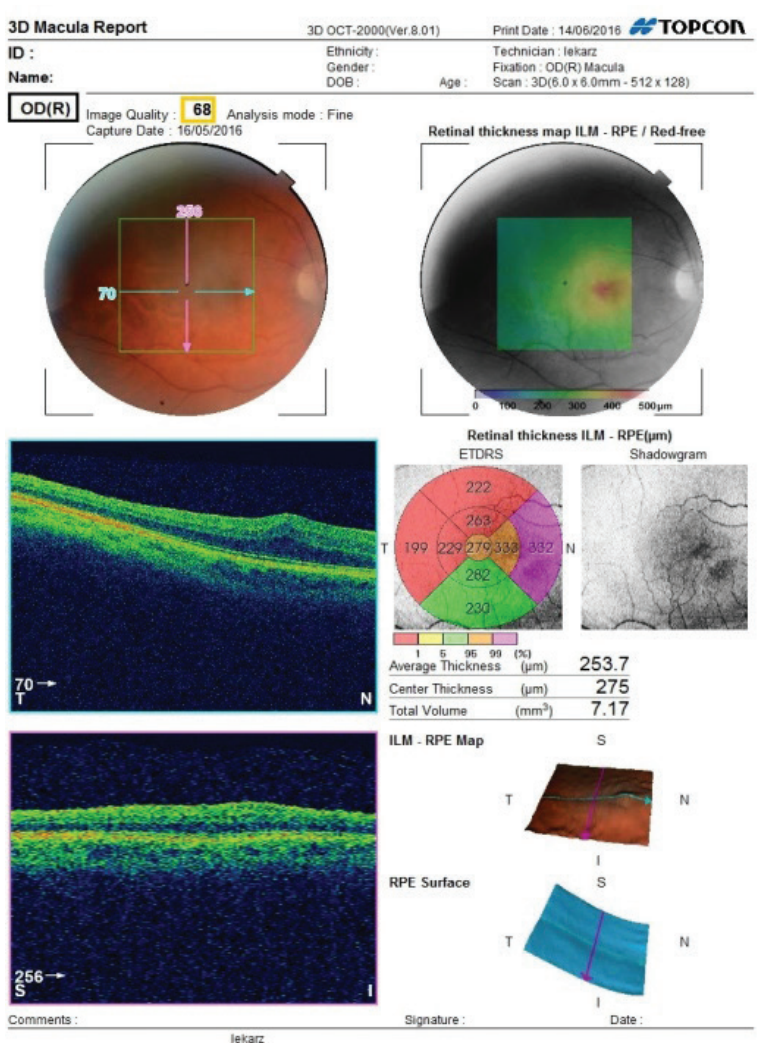

FIGURE 3. Ocular coherence tomography of the macula after vitrectomy in a patient with Terson syndrome

aging examination (CT, computed tomography; MRI, magnetic resonance imaging; angio-CT, angio-computed tomography) providing evidence of intracranial injury, neurological examination of eyes functions should be done. Terson syndrome can be confirmed by ophthalmic examination as fundus examination and ultrasonography of the eyeball [6]. If the view of the fundus is not possible, to determine the degree of vitreous haemorrhage, ultrasonography examination (B-scan) is needed; it can also exclude detachment of the retina [3].

Clinical studies have shown the importance of early CT examination in detecting subarachnoid haemorrhage with simultaneous vitreous haemorrhage. Among a series of positive results of CT examination there were visible hyperdense areas - nodular or crescent - on the surface of the retina. Although these results cannot be illustrated in all patients, (some are poorly visualised), they can suggest the presence of intraocular haemorrhage [9].

Available methods for the treatment of Terson syndrome include conservative treatment and surgery. In cases of minor bleeding into the vitreous cavity or preretinal bleeding spontaneous resorption 
Table 1. Eye, age, gender, interval from incident to vitrectomy, pre-/postoperative best-corrected visual acuity (BCVA), and pre-/postoperative observations of 5 eyes of 3 patients which underwent vitrectomy due to Terson syndrome

\begin{tabular}{|l|c|c|c|c|c|c|c|}
\hline Case & Eye & $\begin{array}{c}\text { Age } \\
\text { (years) }\end{array}$ & Gender & $\begin{array}{c}\text { Interval from incident to } \\
\text { vitrectomy (months) }\end{array}$ & $\begin{array}{c}\text { Preop } \\
\text { BCVA }\end{array}$ & $\begin{array}{c}\text { Pre-/postoperative } \\
\text { observations }\end{array}$ \\
\hline 1 & Right & 33 & Female & 9 & HM & None \\
BCVA
\end{tabular}

$\mathrm{HM}$ - hand movement; CF — counting fingers

often occurs; this can take a few months (mean nine months). In the initial stage of the conservative treatment of lying patients, a higher position of the head is suggested, as well as avoidance of anticoagulants (e.g. warfarin and aspirin).

In our case series vitrectomy was performed in all cases with good visual outcomes (Tab. 1). Vitrectomy is recommended as beneficial in Terson syndrome since the 1970s. Indications for vitrectomy are as follows: long-lasting (4-6 months) haemorrhage, [2, 9], monocular patients, cases of bleeding in the macular region, bleeding with simultaneous retinal detachment, and bilateral and paediatric cases [2]. Kuhn et al. analysed 124 cases from the literature and found good visual outcomes and a relatively low rate of complications. French researchers retrospectively evaluated the efficacy of vitrectomy as a method of treatment of Terson syndrome. They found that the prognosis regarding to the visual acuity depends on many factors, including significant late effects of this syndrome (epiretinal membrane formation), as well as complications of the vitrectomy (nuclear cataract and endophthalmitis). Also, they proved that final visual acuity affects the neurological condition of the patient (damage to brain structures associated with Terson syndrome). [2,9]. The authors of some studies have shown that another factor that influences the prognosis is the age of the patient $[2,4]$.

Gnanaraj et al. found postoperative development of cataract in $36 \%$ of patients in a retrospective study of 25 eyes [9]. We observed cataract preoperatively in two eyes, so cataract extraction was performed before vitrectomy in one case and as a combination with vitrectomy in another case.

Gnanaraj recommended vitrectomy for the eyes with denser vitreous haemorrhages due to more rapid visual recovery in these eyes. He also recommended that funduscopy should be part of the routine examination of patients with intracranial haemorrhage in order to prevent referral delay. Other reports suggest an early vitrectomy in bilateral cases and an observation period of 3-6 months in unilateral cases. In bilateral cases, an early vitrectomy often results in rapid visual rehabilitation that gives the patient a better chance of general recovery $[11,12]$.

A relative contraindication to surgery is low intensity of haemorrhage, which is particularly likely to be spontaneously resorbed. If the patient's clinical condition is severe, the surgery is contraindicated.

An alternative procedure is the use of an argon laser Nd: YAG, which forms a hole in the posterior membrane of the corpus vitreous and facilitates the evacuation of the contents of a haematoma localised preretinal to the corpus vitreous cavity. It helps further resorption of blood in the following few months and finally improves visual acuity $[2,4]$. The authors of some publications do not recommend this method due to the potential development of future retinal folds or epiretinal membrane [3]. Another example of a less invasive method (rarely used in practice) is to apply intravitreally an antibody $\mathrm{IgG}$ anti-RhD. The absorption of the vitreous haemorrhage depends on the degree of haemolysis of erythrocytes. Administration of antibody IgG anti-RhD accelerates this process. $\mathrm{RhD}$ antigens are specific to red blood cells, and their application does not result in so damage to other tissues. This method of treatment can be used only in Rh positive patients, which limits its use [2].

As complications we observed cataract formation, epiretinal membrane, and tractional retinal detachment. The most common complication of Terson syndrome is the formation of epiretinal 
membranes, observed in approximately $27-78 \%$ of cases. Results from clinical trials suggest that in cases of large and long-existing haemorrhage, some retinal damage may be caused by toxic effects of iron from red blood cells [13]. Intraocular bleeding can lead to proliferation of fibroblasts or glial cells. The resulting retinal folds or epiretinal membranes can significantly impair vision after resorption of haemorrhage. Ritland et al. recommend close follow-up in Terson syndrome with consideration of surgical intervention as soon as epiretinal membrane formation is found in ultrasonography, so that proliferative vitreoretinopathy (PVR) can be treated in its early stage [12]. Other late complications include: decline of retinal pigment epithelium, atrophy the optic nerve, macular hole, retinal folds, cysts of the retina, cataract, and PVR $(3,12)$.

There is a clear correlation between morbidity and mortality due to the intracranial haemorrhage (SAH mainly) and the occurrence of intraocular haemorrhage, confirmed with a large number of studies and observations. In the course of Terson syndrome, a vitreous haemorrhage is saddled with 3-9-fold higher mortality than haemorrhage to other places inside the eyeball [3]. Some studies suggest that the presence of blood inside the eye may be a sign of re-bleeding aneurysm [5, 7]. The occurrence of intraocular haemorrhage signals a serious clinical condition of patient's after brain haemorrhage.

\section{CONCLUSIONS}

The occurrence of Terson syndrome is closely correlated with the serious condition of the patient and poor prognosis with regard to the consequenc- es of the increase of intracranial pressure [1]. Although some patients reveal spontaneous resorption of blood in the eye, many require specialised eye care, especially those with bilateral involvement. Performing vitrectomy with BSS as a tamponade in patients with Terson syndrome significantly improves visual acuity, so early diagnosis and surgical treatment are crucial.

\section{REFERENCES}

1. Bin JX, Qian SL. Terson syndrome with no cerebral hemorrhage: A case report. Experimental and Therapeutic Medicine 2014; 7: 251-253.

2. Czaplicka E, Grabska-Liberek I, Rospond I, Kocięcki J. Zespół Tersona — omówienie przypadków klinicznych i postępowania leczniczego. Borgis - Postępy Nauk Medycznych 2013; 12: 901-903.

3. Ou RJr, Talavera F, Charles S, Roy H, Phillpotts B, Yoshizumi M. Terson Syndrome treatment and management. Med Scape 2014; 13: 312-318.

4. Sánchez Ferreiro AV, Muñoz Bellido L. Atypical presentation of Terson syndrome, Presentation of a case. Neurologia 2012; 27: 380-381.

5. Browning D. What you should know about Terson's syndrome. Retinareference 2013; 1: 121.

6. Sang-Hee L, Jeong-Hwan S, Sung-Hee P, Hui W Y, Myoung-Hwan K, Rehabil $A$. Terson syndrome in aneurysmal subarachnoid hemorrhage: A case report. Med Clin 2015; 39: 640-644.

7. Ogawa T, Kitaoka T, Dake Y, Amemiya T. Terson syndrome: a case report suggesting the mechanism of vitreous hemorrhage. Oftalmology 2001; 108: 1654-1656.

8. Gauntt CD, Sherry RG, Kannan C. Terson syndrome with bilateral optic nerve sheath hemorrhage. J Neuroophthalmol 2007; 27: 193-194.

9. Nacef L, Zghal-Mokni I, Allagui I. Indications and results of vitrectomy in Terson syndrome. Tunis Med 2004; 82: 461-464.

10. Kuhn F, Morris R, Witherspoon CD, Mester V. Terson syndrome results of vitectomy and the significance of vitreous hemorrhage in patients with subarachnoid hemorrhage. Ophthalmology 1998; 105: 472-477.

11. Gnanaraj L, Tyagi AK, Cottrell DG, Fetherston TJ, Richardson J, Stannard KP. Referral delay and ocular surgical outcome in Terson syndrome. Retina 2000; 20: 374-377.

12. Ritland JS, Syrdalen P, Eide N, Vatne HO, Overgaard R. Outcome of vitrectomy in patients with Terson syndrome. Acta Ophthalmol Scand 2002; 80: 172-175.

13. Swallow ChE, Tsuruda JS, Digre KB, Glaser M, Davidson Ch, Harnsberger R. Terson syndrome: CT evaluation in 12 patients. AJNR Am J Neuroradiol 1998; 19: 743-747. 